\title{
О новом методе решения одной биологической задачи большой размерности
}

\author{
Г.В. Демиденко (iD $凶$
}

\begin{abstract}
Аннотация: Рассмотрена задача Коши для системы обыкновенных дифференциальных уравнений большой размерности, моделирующей многостадийный синтез вещества. Предложен метод построения приближенного решения задачи Коши. Метод основан на использовании установленных связей между решениями рассматриваемой системы обыкновенных дифференциальных уравнений, уравнения с запаздывающим аргументом и уравнения с частными производными параболического типа. Ключевые слова: система обыкновенных дифференциальных уравнений большой размерности; уравнение с запаздывающим аргументом; уравнение с частными производными.
\end{abstract}

Благодарности: Работа выполнена при поддержке Российского фонда фундаментальных исследований, проект № 18-29-10086.

Для цитирования: Демиденко Г.В. О новом методе решения одной биологической задачи большой размерности. Письма в Вавиловский журнал генетики и селекции. 2020;6(4):185-187. DOI 10.18699/Letters2020-6-23

\section{Original article}

\section{On a new method for solving a biological problem of high dimension}

\section{G.V. Demidenko (iD凶}

Abstract: We consider the Cauchy problem for a system of ordinary differential equations of high dimension that models multistage substance synthesis. A method for constructing an approximate solution to the Cauchy problem is proposed. This method is based on the use of established relationships between solutions to the system of ordinary differential equations under consideration, a delay equation and a partial differential equation of parabolic type.

Key words: system of ordinary differential equations of high dimension; delay equation; partial differential equation.

For citation: Demidenko G.V. On a new method for solving a biological problem of high dimension. Pisma v Vavilovskii Zhurnal Genetiki i Selektsii = Letters to Vavilov Journal of Genetics and Breeding. 2020;6(4):185-187. DOI 10.18699/Letters2020-6-23 (in Russian)

В работе рассматривается проблема приближенного нахождения решения задачи Коши для системы обыкновенных дифференциальных уравнений, моделирующей многостадийный синтез вещества

$$
\begin{gathered}
\frac{d x_{1}}{d t}=-\frac{n-1}{\tau} x_{1}+g\left(t, x_{n}\right), \\
\frac{d x_{j}}{d t}=\frac{n-1}{\tau}\left(x_{j-1}-x_{j}\right), \quad j=2, \ldots, n-1, \\
\frac{d x_{n}}{d t}=\frac{n-1}{\tau} x_{n-1}-\theta x_{n}, \\
\left.x\right|_{t=0}=a,
\end{gathered}
$$

где $x_{i}(t)$ - концентрация вещества на $i$-й стадии, $\tau>0$ - суммарное время протекания $n$ стадий, $\theta \geq 0$ - параметр диссипации. Первое уравнение - закон инициации, последнее уравнение - закон утилизации, промежуточные уравнения описывают динамику процесса синтеза вещества.

Система (1) входит в класс систем дифференциальных уравнений, введенных в работе (Goodwin, 1965) при моделировании различных биологических процессов.

Отметим, что процесс синтеза вещества может иметь сотни тысяч промежуточных стадий. Поэтому при решении задачи (1) исследователь может столкнуться с серьезными трудностями, поскольку ввиду нелинейности функции $g(t, z)$ получение аналитических формул для решений, за 
редким исключением, невозможно, а в силу огромной размерности непосредственное решение (1) на компьютере может быть нереально.

Мы сейчас расскажем о методе построения приближенного решения задачи Коши (1) при $n \gg 1$. Этот метод основан на установленных связях между решениями специальных классов систем обыкновенных дифференциальных уравнений большой размерности, решениями уравнений с запаздывающим аргументом и решениями некоторых уравнений с частными производными.

Вначале отметим, что в задаче синтеза вещества биологов прежде всего интересует концентрация конечного продукта. Поэтому, рассматривая систему (1), нужно уметь достаточно точно вычислять последнюю компоненту решения $x_{n}(t)$ при $n \gg 1$. Но из вида системы вытекает, что ни одним из ее уравнений пренебречь нельзя. Следовательно, при рассмотрении задачи Коши (1) для системы с очень большим числом уравнений возникает «проблема большой размерности»!

Проблема нахождения приближенных значений последней компоненты решения задачи (1) при $n \gg 1$ в случае нулевых начальных данных была решена в (Лихошвай и др., 2004). Ключевым моментом при решении «проблемы большой размерности» для этой системы послужила гипотеза, высказанная В.А. Лихошваем. Суть этой гипотезы заключается в том, что с биологической точки зрения концентрация получаемого вещества $x_{n}(t)$ при $n \gg 1$ приближенно должна описываться решением дифференциального уравнения с запаздывающим аргументом

$$
\frac{d}{d t} y(t)=-\theta y(t)+g(t-\tau, y(t-\tau)) .
$$

Расчеты, проведенные С.И. Фадеевым, подтверждали эту гипотезу. Аналитическое доказательство этой гипотезы было получено автором.

Напомним вкратце идею ее доказательства. Будем неограниченно увеличивать число уравнений системы (1) и при каждом $n$ рассматривать только последнюю компоненту решения задачи Коши. Для простоты будем считать, что в (1) задаются нулевые начальные условия.

Предположим, что

$$
\begin{gathered}
g(t, z) \in C\left(R_{2}\right), \quad|g(t, z)| \leq G \\
\left|g\left(t, z_{1}\right)-g\left(t, z_{2}\right)\right| \leq L\left|z_{1}-z_{2}\right|, \quad z_{1}, z_{2} \in R .
\end{gathered}
$$

Тогда при любом фиксированном $n$ каждая задача (1) однозначно разрешима на полуоси, и мы получим последовательность функций $\left\{x_{n}^{n}(t)\right\}$, состоящую из последних компонент решений серии задач Коши (1) (верхний индекс означает число уравнений в системе, нижний - номер компоненты решения). Из работы (Лихошвай и др., 2004) вытекает следующее утверждение о сходимости $\left\{x_{n}^{n}(t)\right\}$.

Теорема. В случае нулевых начальных данных последовательность $\left\{x_{n}^{n}(t)\right\}$ равномерно сходится на любом отрезке $[0, T], T>\tau$ :

$$
x_{n}^{n}(t) \rightarrow y(t), \quad n \rightarrow \infty .
$$

Предельная функция у $(t)$ является решением начальной задачи для уравнения с запаздывающим аргументом (2):

$$
\begin{gathered}
\frac{d}{d t} y(t)=-\theta y(t)+g(t-\tau, y(t-\tau)), \quad t>\tau, \\
y(t)=0, \quad 0 \leq t \leq \tau, \\
y(\tau+0)=0,
\end{gathered}
$$

при этом имеет место оценка

$$
\max _{t \in[0, T]}\left|x_{n}^{n}(t)-y(t)\right| \leq \frac{c}{n^{\frac{1}{4}}}, \quad n>n_{0},
$$

где константа с > 0 зависит от функции $g(t, z)$, величины T и параметров $\tau, \theta$.

Предельная теорема дает математическое обоснование для приближенного нахождения концентрации получаемого вещества $x_{n}(t)$ при $n \gg 1$ с использованием уравнения с запаздывающим аргументом (2). Именно для этого достаточно приближенно решить начальную задачу (3), при этом, учитывая скорость сходимости (4), можно оценить погрешность аппроксимации

$$
x_{n}(t) \approx y(t), \quad n \gg 1 .
$$

Очевидно, чем большее количество стадий $n$ требуется для получения конечного продукта синтеза, тем точнее можно получить результат, следуя этому методу. Отметим, что при не слишком больших значениях $n$ (до порядка $10^{4}-10^{6}$ ) хорошие результаты можно получить, численно решая задачу Коши (1) с использованием стандартных математических пакетов.

Описанный метод приближенного нахождения концентрации конечного продукта можно обобщить на случай ненулевых начальных данных, но для этого нужно доказать соответствующие предельные теоремы. Можно показать (Демиденко, Мельник, 2010), что при рассмотрении серии задач Коши (1) с ненулевыми начальными условиями справедливы аналоги предельной теоремы. Однако в отличие от нулевых начальных условий здесь возникает интересная особенность, заключающаяся в том, что, вообще говоря, нет равномерной сходимости последовательности $\left\{x_{n}^{n}(t)\right\}$, но сходимость можно гарантировать в пространствах Лебега, при этом предельная функция $y(t)$ будет обобщенным решением дифференциального уравнения (2). Следовательно, вместо классических решений уравнения с запаздывающим аргументом (2) мы будем иметь дело с обобщенными решениями.

Рассмотрим теперь задачу синтеза вещества, связанную с наблюдениями за изменением концентрации вещества на промежуточных стадиях. Оказывается, опираясь на предельные теоремы, построение приближенных решений задачи Коши (1) при $n \gg 1$, можно свести к решению первой краевой задачи для уравнения с частными производными вида

$$
\tau u_{t}+u_{z}-\frac{1}{2(n-1)} u_{z z}=0, \quad t>0, \quad z \in(0,1) .
$$

Для простоты вновь ограничимся рассмотрением задачи Коши (1) при нулевых начальных условиях. Пусть

$$
x(t)=\left(x_{1}(t), \ldots, x_{n}(t)\right)^{T}
$$

ее решение. Рассмотрим гладкую функцию $u(t, z)$ в прямоугольнике 


$$
G=\{t>0, \quad z \in(0,1)\},
$$

непрерывную вплоть до границы. Предположим, что в узлах

$$
z_{k}=\frac{k-1}{n-1}, \quad k=1,2, \ldots, n,
$$

имеет место равенство

Тогда

$$
u\left(t, z_{k}\right)=x_{k}(t) .
$$

$$
u\left(t, z_{1}\right)=u(t, 0)=x_{1}(t), \quad u\left(t, z_{n}\right)=u(t, 1)=x_{n}(t) .
$$

Заметим, что все промежуточные уравнения системы (1) можно записать в виде

$$
\begin{aligned}
\tau u_{t}\left(t, z_{k}\right)= & (n-1)\left(u\left(t, z_{k}-\frac{1}{n-1}\right)-u\left(t, z_{k}\right)\right), \\
& k=2, \ldots, n-1 .
\end{aligned}
$$

Действительно, учитывая (6), имеем

$$
\begin{aligned}
\tau u_{t}\left(t, z_{k}\right) & =\tau \frac{d}{d t} x_{k}(t) \\
u\left(t, z_{k}-\frac{1}{n-1}\right)-u\left(t, z_{k}\right) & =u\left(t, \frac{(k-1)-1}{n-1}\right)-u\left(t, z_{k}\right) \\
= & x_{k-1}(t)-x_{k}(t)
\end{aligned}
$$

Следовательно, $k$-е уравнение системы (1) имеет вид (7).

Используя определение псевдодифференциальных операторов, см., например (Маслов, 1973), уравнения (7) можно переписать в виде

$$
\begin{gathered}
\frac{1}{n-1} u_{t}(t, z)-i \sin \left(\frac{i}{n-1} D_{z}\right) u(t, z)+ \\
2 \sin ^{2}\left(\frac{i}{2(n-1)} D_{z}\right) u(t, z)=0
\end{gathered}
$$

при $z=z_{k}$

Рассмотрим псевдодифференциальное уравнение (8) в прямоугольнике $G$ и поставим для него краевую задачу таким образом, чтобы ее решение на прямых $\left(t, z_{k}\right)$ при $k=1, \ldots, n$ совпадало бы с соответствующими компонента-ми решения задачи Коши (1).

Поскольку $a=0$, то начальные условия, естественно, можно взять нулевыми

$$
u(0, z)=0 .
$$

Выпишем краевые условия при $z=0$ и $z=1$. Из первого уравнения системы (1) имеем

Поэтому

$$
x_{1}(t)=\int_{0}^{t} e^{-\frac{n-1}{\tau}(t-s)} g\left(s, x_{n}(s)\right) d s .
$$

$$
u(t, 0)=\int_{0}^{t} e^{-\frac{n-1}{\tau}(t-s)} g(s, u(s, 1)) d s .
$$

Из последнего уравнения (1) имеем

$$
x_{n}(t)=-\frac{n-1}{\tau} \int_{0}^{t} e^{-\theta(t-s)} x_{n-1}(s) d s .
$$

Поэтому

$$
u(t, 1)=-\frac{n-1}{\tau} \int_{0}^{t} e^{-\theta(t-s)} u\left(s, 1-\frac{1}{n-1}\right) d s .
$$

Учитывая уравнение (8), граничные условия (9)-(11), а также предельную теорему, можно указать краевую задачу, решение которой на прямых $\left.\left(t, z_{k}\right), k=1\right), \ldots, n$ при $n \gg 1$ можно рассматривать как приближенное значение соответствующих компонент решения задачи Коши (1).

Для этого рассмотрим приближения уравнения (8) при $n \rightarrow \infty$.

Первое приближение этого уравнения при $n \rightarrow \infty$, очевидно, имеет вид

$$
\tau u_{t}(t, z)+u_{z}(t, z)=0
$$

Но смешанная краевая задача с условиями (9)-(11) для этого уравнения некорректна.

Выписывая второе приближение уравнения (8), при увеличении $n$ получаем уравнение (5). Ясно, что смешанная краевая задача с условиями (9)-(11) для него корректна. Такую краевую задачу можно рассматривать как задачу, позволяющую строить приближенные решения задачи Коши при $n \gg 1$. Учитывая предельную теорему, в силу которой $x_{n}(t) \approx y(t), n \gg 1$, где $y(t)-$ решение начальной задачи (3), условия (10), (11), очевидно, можно упростить. А полученная первая краевая задача для уравнения (5) легко решается, например, методом Фурье.

В заключение добавлю, что мой интерес к изучению классов систем дифференциальных уравнений, возникающих при моделировании биологических процессов, а также к уравнениям с запаздывающим аргументом возник благодаря кипучей деятельности Виталия Александровича Лихошвая в наших совместных интеграционных проектах. Я благодарен ему за это.

\section{Список литературы / References}

Демиденко Г.В., Мельник И.А. Об одном способе аппроксимации решений дифференциальных уравнений с запаздывающим аргументом. Сиб. мат. журн. 2010;51(3):528-546.

[Demidenko G.V., Mel'nik I.A. On a method of approximation of solutions to delay differential equations. Siberian Mathem. Journal. 2010;51(3):419-434. DOI 10.1007/s11202-010-0043-2.]

Лихошвай В.А., Фадеев С.И., Демиденко Г.В., Матушкин Ю.Г. Моделирование уравнением с запаздывающим аргументом многостадийного синтеза без ветвления. Сиб. журн. индустр. мат. 2004;7(1):73-94.

[Likhoshvai' V.A., Fadeev S.I., Demidenko G.V., Matushkin Yu.G. Modeling multistage synthesis without branching by a delay equation. Sibirskii Zhurnal Industrial'noi Matematiki. 2004;7(1):73-94. (in Russian)] Маслов В.П. Операторные методы. М.: Наука, 1973.

[Maslov V.P. Operational methods. Moscow: Mir Publishers, 1976.]

Goodwin B.C. Oscillatory behavior of enzymatic control processes. Adv. Enzyme Reg. 1965;3:425-439. DOI 10.1016/0065-2571(65)90067-1. 